\title{
ZSCAN25 and CYP2E1 Polymorphisms is Risk Factors for Ischemic Stroke in a Chinese Han Population: A Case Control Study
}

\section{Haozheng Yuan}

The Second Affiliated Hospital of Xi'an Jiaotong University

\section{Pei Fan}

The Second Affiliated Hospital of Xi'an Jiaotong University

\section{Li Yao}

The Second Affiliated Hospital of Xi'an Jiaotong University

\section{Yuying LV}

The Second Affiliated Hospital of Xi'an Jiaotong University

\section{Haidong Wei}

The Second Affiliated Hospital of Xi'an Jiaotong University

Juan Zheng

The Second Affiliated Hospital of Xi'an Jiaotong University

Xinsheng Han ( $\nabla$ xinsheng_han@163.com )

The Second Affiliated Hospital of Xi'an Jiaotong University

\section{Research}

Keywords: ischemic stroke, ZSCAN25, CYP2E1, genetic variants, susceptibility

Posted Date: June 10th, 2020

DOI: https://doi.org/10.21203/rs.3.rs-33406/v1

License: (c) (i) This work is licensed under a Creative Commons Attribution 4.0 International License. Read Full License 


\section{Abstract}

Background: We aimed to explore the relationship between ZSCAN25 and CYP2E1 polymorphisms and Ischemic stroke (IS) susceptibility among a Chinese Han population.

Methods: We enrolled 477 patients with IS and 480 age- and sex- matched health controls. Genotyping of the ZSCAN25 rs10242455, CYP2E1 rs2070672 and rs2515641 were performed by Agena MassARRAY platform. Odds ratio (OR) and $95 \%$ confidence interval $(\mathrm{Cl})$ were calculated by logistic regression analysis.

Results: Rs10242455 (OR $=0.56,95 \% \mathrm{Cl}: 0.34-0.93, p=0.024)$ was associated with a reduced IS susceptibility, while rs2070672 (OR $=1.40,95 \% \mathrm{Cl}: 1.12-1.75, p=0.003)$ and rs2515641 (OR = 1.29, 95\% Cl: $1.01-1.64, p=0.041)$ with an increased IS occurrence. Rs2070672 was observed to correlate with IS risk (OR $=4.06, p=0.038)$ at age $>64$ years, and rs10242455 (OR $=0.45, p=0.021)$ and rs2070672 (OR = 3.28, $p=0.024)$ affected IS risk in males. In addition, rs10242455 (OR $=1.72, p=0.014)$ was significantly associated with hypertension in IS patients.

Conclusion: Our study firstly found that rs10242455 in ZSCAN25, rs2070672 and rs2515641 in CYP2E1 were associated with the occurrence of IS in a Chinese Han population.

\section{Introduction}

Ischemic stroke (IS) is a common serious cerebrovascular disease that causes mortality and disability worldwide. Globally, there were almost $71 \%$ of 25.7 million IS survivors, $51 \%$ of 6.5 million deaths from IS, and $67 \%$ of 10.3 million new IS patients ${ }^{[1]}$. In China, stroke had been identified as the leading causes of death and disability-adjusted life-years at the national level in $2017^{[2]}$. The pathogenesis of IS still is unclear, but increasing evidence suggests that its etiology affected by various environmental and genetic factors. Observational studies have shown that age, sex, cardiovascular diseases, dyslipidemia, and hypertension advanced age are considered to be risk factors of IS ${ }^{[3,4]}$. Moreover, genetic factors were reported to play a role in the pathophysiological process of IS. Several studies have pointed to genetic variants, such as polymorphisms in TNIP1, MMP2, AHSG that may contribute to the susceptibility of IS ${ }^{[5-7]}$.

Zinc finger and SCAN domain containing 25 (ZSCAN25) gene, also known ZNF498, encodes a protein that bears some similarity to zinc finger proteins, which are involved in DNA binding and protein-protein interactions ${ }^{[8]}$. Seidel K et al. found that zinc finger protein might be involved in neuroprotection and was dysregulated in stroke ${ }^{[9]}$. Evidence suggested that ZNF650 variants were associated with IS and was independent of traditional cardiovascular risk factors ${ }^{[10]}$. ZNF208 polymorphisms were proved to associate with risk of IS ${ }^{[11]}$. So far, little is known about the detail of the ZNF498 gene on IS.

Cytochrome P450s (CYPs) are a large family of monooxygenase enzymes which are responsible for the oxidative metabolism of endogenous and exogenous compounds ${ }^{[12]}$. CYP-derived lipid mediator has important functions in cerebral vascular function as well as their role in ischemic stroke ${ }^{[13]}$. CYP2E1 gene encodes a member of the cytochrome P450 superfamily of enzymes, which catalyze the metabolism and synthesis of cholesterol, steroids and other lipids ${ }^{[14]}$. CYP2E1 gene was reported to have an important effect on various diseases including coronary artery lesions,

atherosclerosis and diabetes ${ }^{[15-17]}$. These studies suggested that $C Y P 2 E 1$ might contribute to the pathogenesis of IS. To date, many studies have investigated the association of $C Y P 2 E 1$ genetic variants with IS ${ }^{[18,19]}$, but not in Chinese Han population.

Here, our study explored the relationship between ZSCAN25 and CYP2E1 polymorphisms and susceptibility to IS among a Chinese Han population. We also estimated the probable effect of the confounding risk factors, including age, sex, 


\section{Methods}

\section{Subject Recruitment}

We enrolled 477 patients diagnosed with IS at the Department of Neurology of The Second Affiliated Hospital of Xi'an Jiaotong University. All of the enrolled IS patients were confirmed by combining the medical history and neuroimaging data (CT, MRI or MR angiography). Patients with hemorrhagic stroke, brain injury, stroke caused by other causes, tumors, other brain disease, inflammatory disorders or serious chronic diseases were excluded from this study. Four hundred and eighty age, sex, race and geographical area matched unrelated healthy controls were included, who were recruited from the health checkup center in the same hospital. The controls who had a history of IS, hypertension and coronary heart disease (CHD), cerebrovascular disease, arterial vascular disease, other neurological disease or inflammatory disorders were excluded. Demographic and clinical information, including age, sex, history of hypertension and CHD, total protein, total bilirubin, triglyceride, total cholesterol, high-density lipoprotein cholesterol (HDL-C), low-density lipoprotein cholesterol (LDL-C), hemoglobin, total leukocyte count (TLC), and platelets (PLT) of IS patients and controls were obtained from questionnaires and medical records. This study protocol was in accordance with the Declaration of Helsinki and permitted by the ethics committee of The Second Affiliated Hospital of Xi'an Jiaotong University, and written informed consent was obtained from all subjects.

\section{DNA extraction and SNP Genotyping}

Five $\mathrm{mL}$ of peripheral whole blood was collected in EDTA tubes vacutainer and stored at $4{ }^{\circ} \mathrm{C}$ before DNA isolation. The genomic DNA was extracted using GoldMag DNA Purification Kit (GoldMag Co. Ltd., Xi'an, China). The concentration and purity of DNA were determined by Nano Drop 2000 (Thermo Scientific, USA).

Three SNPs were selected with an $r^{2}>0.8$ by Haploview and with minor allele frequency (MAF) $>0.05$ from 1000 Genomes Project data. Genotyping of the ZSCAN25 rs10242455, CYP2E1 rs2070672 and rs2515641 polymorphisms were analyzed by Agena MassARRAY platform (Agena, San Diego, CA, USA) in double-blind fashion. Detail primer sequences were presented in Supplementary Table 1. About 10\% samples were randomly selected for repeat assays and the results were $100 \%$ concordant.

\section{Data Analyses}

The difference of demographic and clinical data between IS cases and health controls was tested by $\chi^{2}$ test (categorical variables) or the Student's t test (continuous variables). Hardy-Weinberg equilibrium (HWE) was used to assess the genotype frequencies in the control subjects by Pearson's $\chi^{2}$ test. The allele and genotype frequencies between the two groups were analyzed by $\chi^{2}$ test. Odds ratio (OR) and 95\% confidence interval (CI) for adjustment of age and sex were calculated by logistic regression analysis for the association of ZSCAN25 and CYP2E1 polymorphisms with IS susceptibility. Stratification analysis was performed to estimate potential effect of the confounding risk factors, including age, sex, hypertension and coronary heart disease on the association. Analysis of Variance (ANOVA) was applied for the relationship between genotypes and blood lipid parameters. Data analysis was performed with SPSS 20.0 software (SPSS Inc., Chicago, IL) and PLINK 2.1.7 software. $p$ value was two-tailed and $<0.05$ to be statistically significant.

\section{Results}

\section{Characteristics of Study Population}


Baseline demographic and clinical features were shown in Table 1. A total of 477 IS (316 males and 161 females) patients and 480 healthy controls (313 males and 167 females) were recruited for the study. The mean age was $64.13 \pm$ 10.82 years for IS patients and $63.69 \pm 6.69$ years for the control subjects. The distribution of age and gender was no significant differences between two groups ( $p=0.443$ and $p=0.735$, respectively). There were no significant differences between cases and controls in levels of triglyceride, HDL-C, hemoglobin, PLT; but significant differences for the levels of total protein, total bilirubin, total cholesterol, LDL-C and TLC were found. Cases included 340 patients (71.3\%) with hypertension and 103 patients $(21.6 \%)$ with coronary heart disease.

Table 1

Characteristics of patients with IS patients and controls

\begin{tabular}{|c|c|c|c|}
\hline Variable & Cases $(n=477)$ & Controls $(n=480)$ & $p$ \\
\hline Age, year (mean \pm SD) & $64.13 \pm 10.82$ & $63.69 \pm 6.69$ & 0.443 \\
\hline Gender,(male/female), n & $316 / 161$ & $313 / 167$ & 0.735 \\
\hline Total protein $(\mathrm{g} / \mathrm{L})$ & $53.50 \pm 24.36$ & $66.40 \pm 18.33$ & $<0.001$ \\
\hline Total bilirubin $(\mu \mathrm{mol} / \mathrm{L})$ & $12.35 \pm 7.55$ & $14.85 \pm 6.99$ & 0.001 \\
\hline Triglyceride (mmol/L) & $1.61 \pm 0.96$ & $1.80 \pm 1.40$ & 0.080 \\
\hline Total cholesterol (mmol/L) & $3.94 \pm 1.02$ & $5.50 \pm 7.90$ & 0.020 \\
\hline HDL-C (mmol/L) & $1.18 \pm 0.32$ & $1.19 \pm 0.30$ & 0.696 \\
\hline LDL-C (mmol/L) & $1.87 \pm 0.60$ & $2.69 \pm 0.73$ & $<0.001$ \\
\hline Hemoglobin $(\mathrm{g} / \mathrm{L})$ & $122.87 \pm 45.41$ & $129.79 \pm 37.40$ & 0.073 \\
\hline $\operatorname{TLC}\left(10^{9} / \mathrm{L}\right)$ & $7.84 \pm 8.27$ & $5.72 \pm 1.40$ & $<0.001$ \\
\hline $\operatorname{PLT}\left(10^{9} / \mathrm{L}\right)$ & $152.89 \pm 85.51$ & $150.18 \pm 94.77$ & 0.763 \\
\hline Hypertension, $n$ & $340(71.3 \%)$ & & \\
\hline Coronary heart disease, $\mathrm{n}$ & $103(21.6 \%)$ & & \\
\hline \multicolumn{4}{|c|}{$\begin{array}{l}\text { IS; ischemic stroke; HDL-C, high-density lipoprotein cholesterol; LDL-C, low-density lipoprotein cholesterol; TLC, tota } \\
\text { leukocyte count; PLT, platelets. }\end{array}$} \\
\hline
\end{tabular}

\section{Association of ZSCAN25 and CYP2E1 polymorphism with IS risk.}

Three selected SNPs were successfully genotyped, and the success rate of genotyping was $>99.2 \%$. All genotype distribution of the studied SNPs in controls was in HWE ( $p>0.05$, Supplementary Table 2$)$. The allelic and genotype frequencies for selected SNPs between cases and controls were shown in Table 2. The results of association analysis showed that ZSCAN25 rs10242455 was a protect factor for IS susceptibility; while rs2070672 and rs2515641 in CYP2E1 conferred to the increased risk for IS occurrence. For rs 10242455, G allele (G vs A: OR $=0.81,95 \% \mathrm{Cl}: 0.66-0.99, p=$ 0.036; and $\mathrm{AA}+\mathrm{AG}+\mathrm{GG}: \mathrm{OR}=0.80,95 \% \mathrm{Cl}: 0.66-0.98, p=0.033$ ) and $\mathrm{GG}$ genotype (GG vs $\mathrm{AA}: \mathrm{OR}=0.56,95 \% \mathrm{Cl}: 0.34-$ $0.93, p=0.024$; and GG vs AA-AG: OR $=0.60,95 \% \mathrm{Cl}: 0.36-0.97, p=0.039)$ were related to the reduced IS risk. The significant association of rs2070672 polymorphism with IS susceptibility was found under the allele $(\mathrm{OR}=1.40,95 \% \mathrm{Cl}$ : $1.12-1.75, p=0.003)$, genotype (OR = 2.81, 95\% Cl: $1.37-5.77, p=0.005)$, dominant (OR = 1.38, 95\% Cl: $1.06-1.80, p=$ $0.017)$, recessive $(\mathrm{OR}=2.58,95 \% \mathrm{Cl}: 1.26-5.27, p=0.009)$, additive $(\mathrm{OR}=1.41,95 \% \mathrm{Cl}: 1.12-1.77, p=0.003)$ models. In 
addition, we also found that rs2515641 was increased IS occurrence ( $\mathrm{T}$ vs C: OR = 1.27, 95\% Cl: $1.00-1.62, p=0.046$; and CC + CT + TT: OR = 1.29, 95\% Cl: 1.01-1.64, $p=0.041)$. 
Table 2

Association between ZSCAN25 and CYP2E1 polymorphisms and IS risk

\begin{tabular}{|c|c|c|c|c|c|c|}
\hline \multirow[t]{2}{*}{ SNP ID } & \multirow[t]{2}{*}{ Model } & \multirow[t]{2}{*}{ Genotype } & \multirow[t]{2}{*}{ Case } & \multirow[t]{2}{*}{ Control } & \multicolumn{2}{|c|}{ Adjusted by age and gender } \\
\hline & & & & & OR $(95 \% \mathrm{Cl})$ & $\mathrm{p}$ \\
\hline \multirow{10}{*}{$\begin{array}{l}\text { ZSCAN25 } \\
\text { rs10242455 }\end{array}$} & Allele & $A$ & 705 & 668 & 1 & \\
\hline & & G & 249 & 292 & $0.81(0.66-0.99)$ & 0.036 \\
\hline & Genotype & AA & 256 & 233 & 1 & \\
\hline & & $A G$ & 193 & 202 & $0.87(0.67-1.14)$ & 0.305 \\
\hline & & GG & 28 & 45 & $0.56(0.34-0.93)$ & 0.024 \\
\hline & Dominant & AA & 256 & 233 & 1 & \\
\hline & & AG-GG & 221 & 247 & $0.81(0.63-1.05)$ & 0.112 \\
\hline & Recessive & AA-AG & 449 & 435 & 1 & \\
\hline & & GG & 28 & 45 & $0.60(0.36-0.97)$ & 0.039 \\
\hline & Log-additive & - & - & - & $0.80(0.66-0.98)$ & 0.033 \\
\hline \multirow{10}{*}{$\begin{array}{l}\text { CYP2E1 } \\
\text { rs2070672 }\end{array}$} & Allele & $A$ & 734 & 787 & 1 & \\
\hline & & G & 220 & 169 & $1.40(1.12-1.75)$ & 0.003 \\
\hline & Genotype & AA & 284 & 320 & 1 & \\
\hline & & $A G$ & 166 & 147 & $1.28(0.97-1.68)$ & 0.082 \\
\hline & & GG & 27 & 11 & $2.81(1.37-5.77)$ & 0.005 \\
\hline & Dominant & AA & 284 & 320 & 1 & \\
\hline & & AG-GG & 193 & 125 & $1.38(1.06-1.80)$ & 0.017 \\
\hline & Recessive & $A A-A G$ & 450 & 467 & 1 & \\
\hline & & GG & 27 & 11 & $2.58(1.26-5.27)$ & 0.009 \\
\hline & Log-additive & - & - & - & $1.41(1.12-1.77)$ & 0.003 \\
\hline \multirow{7}{*}{$\begin{array}{l}\text { CYP2E1 } \\
\text { rs2515641 }\end{array}$} & Allele & C & 762 & 805 & 1 & \\
\hline & & $\mathrm{T}$ & 182 & 151 & $1.27(1.00-1.62)$ & 0.046 \\
\hline & Genotype & $\mathrm{CC}$ & 307 & 336 & 1 & \\
\hline & & $\mathrm{CT}$ & 148 & 133 & $1.22(0.92-1.62)$ & 0.164 \\
\hline & & TT & 17 & 9 & $2.10(0.92-4.79)$ & 0.078 \\
\hline & Dominant & $\mathrm{CC}$ & 307 & 336 & 1 & \\
\hline & & CT-TT & 165 & 142 & $1.28(0.97-1.68)$ & 0.080 \\
\hline
\end{tabular}

IS; ischemic stroke; SNP, single nucleotide polymorphism; OR, odds ratio; 95\% Cl, 95\% confidence interval. $p$ values were calculated by logistic regression analysis with adjustments for age and gender.

Bold indicate that $p<0.05$ means the data is statistically significant. 


\begin{tabular}{|llllll|}
\hline \multicolumn{1}{|l}{ Recessive } & CC-CT & 455 & 469 & 1 & \\
\cline { 2 - 5 } & TT & 17 & 9 & $1.98(0.87-4.49)$ & 0.103 \\
\hline Log-additive & - & - & - & $1.29(1.01-1.64)$ & 0.041 \\
\hline IS; ischemic stroke; SNP, single nucleotide polymorphism; OR, odds ratio; 95\% Cl, 95\% confidence interval. \\
\hline$p$ values were calculated by logistic regression analysis with adjustments for age and gender. \\
\hline Bold indicate that $p<0.05$ means the data is statistically significant.
\end{tabular}

\section{Stratification analysis by age and sex for the association of ZSCAN25 and CYP2E1 variants with IS risk}

Considering age and sex as a potential risk factor for IS, stratification analysis by age and sex was performed to estimate potential effect of age and gender on the association between ZSCAN25 and CYP2E1 variants and IS risk (Table 3). Among the subgroup with age > 64 years, CYP2E1 rs2070672 GG genotype was observed to correlate with the risk of IS compared with AA genotype (OR $=4.06,95 \% \mathrm{Cl}: 1.08-15.26, p=0.038)$ or AA-AG genotype $(\mathrm{OR}=3.90,95 \% \mathrm{Cl}$ : $1.05-$ $14.55, p=0.043)$. In the subgroup at age $\leq 64$ years, ZSCAN25 rs10242455 also had a protective effect on IS susceptibility (G vs A: OR=0.75, 95\% Cl: 0.57-0.98, $p=0.035$; and AA+ AG + GG: OR=0.74, 95\% Cl: 0.55-0.99, $p=0.045$ ). In addition, we observed an increased IS risk for CYP2E1 rs2070672 under the allele (OR=1.41, 95\% Cl: 1.05-1.90, $p=$ $0.023)$, dominant $(\mathrm{OR}=1.49,95 \% \mathrm{Cl}: 1.02-2.17, p=0.038)$, and additive (OR $=1.46,95 \% \mathrm{Cl}: 1.06-2.02, p=0.021)$ models at age $\leq 64$ years. 
Table 3

Association between ZSCAN25 and CYP2E1 polymorphisms and IS risk according to the stratification by gender and age $\begin{array}{lllllllllll}\text { SNP ID } & \text { Model } & \text { Genotype } & \text { Case } & \text { Control } & \underset{(95 \% \mathrm{Cl})}{\mathrm{OR}} & p & \text { Case } & \text { Control } & \begin{array}{l}\mathrm{OR} \\ (95 \% \mathrm{Cl})\end{array} & p\end{array}$

\begin{tabular}{|c|c|c|c|c|c|c|c|c|c|c|}
\hline \multicolumn{3}{|l|}{ Age } & \multicolumn{4}{|c|}{$>64$ years } & \multicolumn{4}{|c|}{$\leq 64$ years } \\
\hline ZSCAN25 & Allele & $A$ & 330 & 270 & 1 & & 375 & 398 & 1 & \\
\hline \multirow[t]{9}{*}{ rs10242455 } & & $G$ & 128 & 120 & $\begin{array}{l}0.87 \\
(0.65- \\
1.17)\end{array}$ & 0.368 & 121 & 172 & $\begin{array}{l}0.75 \\
(0.57- \\
0.98)\end{array}$ & 0.035 \\
\hline & Genotype & AA & 118 & 96 & 1 & & 138 & 137 & 1 & \\
\hline & & $A G$ & 94 & 78 & $\begin{array}{l}0.90 \\
(0.59- \\
1.38)\end{array}$ & 0.639 & 99 & 142 & $\begin{array}{l}0.74 \\
(0.50- \\
1.08)\end{array}$ & 0.113 \\
\hline & & GG & 17 & 21 & $\begin{array}{l}0.58 \\
(0.28- \\
1.21)\end{array}$ & 0.148 & 11 & 24 & $\begin{array}{l}0.54 \\
(0.25- \\
1.18)\end{array}$ & 0.125 \\
\hline & Dominant & $\mathrm{AA}$ & 118 & 96 & 1 & & 138 & 137 & 1 & \\
\hline & & AG-GG & 111 & 99 & $\begin{array}{l}0.83 \\
(0.56- \\
1.25)\end{array}$ & 0.376 & 110 & 148 & $\begin{array}{l}0.71 \\
(0.49- \\
1.02)\end{array}$ & 0.062 \\
\hline & Recessive & $A A-A G$ & 212 & 174 & 1 & & 237 & 261 & 1 & \\
\hline & & GG & 17 & 21 & $\begin{array}{l}0.61 \\
(0.30- \\
1.24)\end{array}$ & 0.170 & 11 & 24 & $\begin{array}{l}0.62 \\
(0.29- \\
1.33)\end{array}$ & 0.221 \\
\hline & $\begin{array}{l}\text { Log- } \\
\text { additive }\end{array}$ & - & - & - & $\begin{array}{l}0.82 \\
(0.60- \\
1.11)\end{array}$ & 0.199 & - & - & $\begin{array}{l}0.74 \\
(0.55- \\
0.99)\end{array}$ & 0.045 \\
\hline CYP2E1 & Allele & $A$ & 355 & 321 & 1.00 & & 379 & 466 & 1 & \\
\hline \multirow[t]{6}{*}{ rs2070672 } & & $\mathrm{G}$ & 103 & 67 & $\begin{array}{l}1.39 \\
(0.99- \\
1.96)\end{array}$ & 0.059 & 117 & 102 & $\begin{array}{l}1.41 \\
(1.05- \\
1.90)\end{array}$ & 0.023 \\
\hline & Genotype & $A A$ & 139 & 130 & 1 & & 145 & 190 & 1 & \\
\hline & & $A G$ & 77 & 61 & $\begin{array}{l}1.13 \\
(0.73- \\
1.75)\end{array}$ & 0.586 & 89 & 86 & $\begin{array}{l}1.41 \\
(0.95- \\
2.08)\end{array}$ & 0.086 \\
\hline & & GG & 13 & 3 & $\begin{array}{l}4.06 \\
(1.08- \\
15.26)\end{array}$ & 0.038 & 14 & 8 & $\begin{array}{l}2.43 \\
(0.93- \\
6.32)\end{array}$ & 0.070 \\
\hline & Dominant & AA & 139 & 130 & 1 & & 145 & 190 & 1 & \\
\hline & & AG-GG & 90 & 64 & $\begin{array}{l}1.27 \\
(0.83- \\
1.93)\end{array}$ & 0.275 & 103 & 94 & $\begin{array}{l}1.49 \\
(1.02- \\
2.17)\end{array}$ & 0.038 \\
\hline
\end{tabular}

IS; ischemic stroke; SNP, single nucleotide polymorphism; OR, odds ratio; 95\% Cl, 95\% confidence interval. $p$ values were calculated by logistic regression analysis with adjustments for age and gender.

Bold indicate that $p<0.05$ means the data is statistically significant. 


\begin{tabular}{|c|c|c|c|c|c|c|c|c|c|c|}
\hline SNP ID & Model & Genotype & Case & Control & $\begin{array}{l}\text { OR } \\
(95 \% \mathrm{Cl})\end{array}$ & $p$ & Case & Control & $\begin{array}{l}\text { OR } \\
(95 \% \mathrm{Cl})\end{array}$ & $p$ \\
\hline & Recessive & AA-AG & 216 & 191 & 1 & & 234 & 276 & 1 & \\
\hline & & GG & 13 & 3 & $\begin{array}{l}3.90 \\
(1.05- \\
14.55)\end{array}$ & 0.043 & 14 & 8 & $\begin{array}{l}2.15 \\
(0.83- \\
5.55)\end{array}$ & 0.113 \\
\hline & $\begin{array}{l}\text { Log- } \\
\text { additive }\end{array}$ & - & - & - & $\begin{array}{l}1.36 \\
(0.94- \\
1.97)\end{array}$ & 0.098 & - & - & $\begin{array}{l}1.46 \\
(1.06- \\
2.02)\end{array}$ & 0.021 \\
\hline Sex & & & \multicolumn{4}{|l|}{ Male } & \multicolumn{4}{|c|}{ Female } \\
\hline ZSCAN25 & Allele & A & 474 & 446 & 1 & & 231 & 222 & 1 & \\
\hline \multirow[t]{9}{*}{ rs10242455 } & & G & 158 & 180 & $\begin{array}{l}0.83 \\
(0.64- \\
1.06)\end{array}$ & 0.133 & 91 & 112 & $\begin{array}{l}0.78 \\
(0.56- \\
1.09)\end{array}$ & 0.144 \\
\hline & Genotype & AA & 172 & 162 & 1 & & 84 & 71 & 1 & \\
\hline & & AG & 130 & 122 & $\begin{array}{l}1.00 \\
(0.72- \\
1.39)\end{array}$ & 0.983 & 63 & 80 & $\begin{array}{l}0.66 \\
(0.42- \\
1.05)\end{array}$ & 0.077 \\
\hline & & GG & 14 & 29 & $\begin{array}{l}0.45 \\
(0.23- \\
0.89)\end{array}$ & 0.021 & 14 & 16 & $\begin{array}{l}0.71 \\
(0.32- \\
1.56)\end{array}$ & 0.391 \\
\hline & Dominant & AA & 172 & 162 & 1 & & 84 & 71 & 1 & \\
\hline & & $A G-G G$ & 144 & 151 & $\begin{array}{l}0.9 \\
(0.66- \\
1.23)\end{array}$ & 0.501 & 77 & 96 & $\begin{array}{l}0.67 \\
(0.43- \\
1.04)\end{array}$ & 0.072 \\
\hline & Recessive & $A A-A G$ & 302 & 284 & 1 & & 147 & 151 & 1 & \\
\hline & & GG & 14 & 29 & $\begin{array}{l}0.45 \\
(0.23- \\
0.87)\end{array}$ & 0.018 & 14 & 16 & $\begin{array}{l}0.86 \\
(0.41- \\
1.84)\end{array}$ & 0.705 \\
\hline & $\begin{array}{l}\text { Log- } \\
\text { additive }\end{array}$ & - & - & - & $\begin{array}{l}0.82 \\
(0.64- \\
1.06)\end{array}$ & 0.129 & - & - & $\begin{array}{l}0.76 \\
(0.54- \\
1.07)\end{array}$ & 0.120 \\
\hline \multirow{4}{*}{$\begin{array}{l}\text { CYP2E1 } \\
\text { rs2070672 }\end{array}$} & Allele & A & 495 & 518 & 1.00 & & 239 & 269 & 1 & \\
\hline & & G & 137 & 104 & $\begin{array}{l}1.38 \\
(1.04- \\
1.83)\end{array}$ & 0.026 & 83 & 65 & $\begin{array}{l}1.44 \\
(0.99- \\
2.08)\end{array}$ & 0.053 \\
\hline & Genotype & AA & 194 & 212 & 1 & & 90 & 108 & 1 & \\
\hline & & AG & 107 & 94 & $\begin{array}{l}1.24 \\
(0.89- \\
1.75)\end{array}$ & 0.207 & 59 & 53 & $\begin{array}{l}1.33 \\
(0.84- \\
2.13)\end{array}$ & 0.225 \\
\hline
\end{tabular}

IS; ischemic stroke; SNP, single nucleotide polymorphism; OR, odds ratio; $95 \% \mathrm{Cl}, 95 \%$ confidence interval. $p$ values were calculated by logistic regression analysis with adjustments for age and gender.

Bold indicate that $p<0.05$ means the data is statistically significant. 


\begin{tabular}{|c|c|c|c|c|c|c|c|c|c|c|}
\hline SNP ID & Model & Genotype & Case & Control & $\begin{array}{l}\text { OR } \\
(95 \% \mathrm{Cl})\end{array}$ & $p$ & Case & Control & $\begin{array}{l}\text { OR } \\
(95 \% \mathrm{Cl})\end{array}$ & $p$ \\
\hline & & GG & 15 & 5 & $\begin{array}{l}3.28 \\
(1.17- \\
9.21)\end{array}$ & 0.024 & 12 & 6 & $\begin{array}{l}2.37 \\
(0.85- \\
6.59)\end{array}$ & 0.098 \\
\hline & Dominant & $\mathrm{AA}$ & 194 & 212 & 1 & & 80 & 108 & 1 & \\
\hline & & AG-GG & 122 & 99 & $\begin{array}{l}1.35 \\
(0.97- \\
1.87)\end{array}$ & 0.076 & 71 & 59 & $\begin{array}{l}1.44 \\
(0.92- \\
2.25)\end{array}$ & 0.109 \\
\hline & Recessive & AA-AG & 301 & 306 & 1 & & 149 & 161 & 1 & \\
\hline & & GG & 15 & 5 & $\begin{array}{l}3.05 \\
(1.10- \\
8.51)\end{array}$ & 0.033 & 12 & 6 & $\begin{array}{l}2.14 \\
(0.78- \\
5.85)\end{array}$ & 0.140 \\
\hline & $\begin{array}{l}\text { Log- } \\
\text { additive }\end{array}$ & - & - & - & $\begin{array}{l}1.40 \\
(1.05- \\
1.86)\end{array}$ & 0.024 & - & - & $\begin{array}{l}1.42 \\
(0.98- \\
2.06)\end{array}$ & 0.060 \\
\hline \multicolumn{11}{|c|}{ IS; ischemic stroke; SNP, single nucleotide polymorphism; OR, odds ratio; $95 \% \mathrm{Cl}, 95 \%$ confidence interval. } \\
\hline \multicolumn{11}{|c|}{$p$ values were calculated by logistic regression analysis with adjustments for age and gender. } \\
\hline \multicolumn{11}{|c|}{ Bold indicate that $p<0.05$ means the data is statistically significant. } \\
\hline
\end{tabular}

Stratified by sex, ZSCAN25 rs10242455 GG genotype had the protective effect on IS risk in males compared with AA genotype (OR $=0.45,95 \% \mathrm{Cl}: 0.23-0.89, p=0.021)$ or $\mathrm{AA}-\mathrm{AG}$ genotype $(\mathrm{OR}=0.45,95 \% \mathrm{Cl}: 0.23-0.87, p=0.018)$, but not females. The contribution of CYP2E1 rs2070672 to the increased IS occurrence was observed for various genetic models ( $\mathrm{G}$ vs $\mathrm{A}: \mathrm{OR}=1.38,95 \% \mathrm{Cl}: 1.04-1.83, p=0.026 ; \mathrm{GG}$ vs $\mathrm{AA}: \mathrm{OR}=3.28,95 \% \mathrm{Cl}: 1.17-9.21, p=0.024 ; \mathrm{GG}$ vs $\mathrm{AA}-\mathrm{AG}: \mathrm{OR}=$ $3.05,95 \% \mathrm{Cl}: 1.10-8.51, p=0.033$; and $\mathrm{AA}+\mathrm{AG}+\mathrm{GG}: \mathrm{OR}=1.40,95 \% \mathrm{Cl}: 1.05-1.86, p=0.024)$ in males.

\section{Association of ZSCAN25 and CYP2E1 variants with hypertension and CHD in IS patients}

We next evaluated the association of ZSCAN25 and CYP2E1 variants with hypertension and CHD in IS patients (Table 4). When IS parents were divided into two subgroups by with hypertension or without hypertension, ZSCAN25 rs 10242455 was significantly associated with hypertension in IS patients (AG vs AA: OR $=1.72,95 \%$ Cl: $1.12-2.64, p=0.014$ and AGGG vs $A A: O R=1.55,95 \% \mathrm{Cl}: 1.03-2.32, p=0.035)$. Moreover, to evaluate the combined effect of IS and CHD, patients were divided into two groups by with $\mathrm{CHD}$ or without CHD. However, there was not significantly associated with $\mathrm{CHD}$ in IS patients. 
Table 4

Association between ZSCAN25 rs10242455 and IS patients with and without hypertension or CHD

\begin{tabular}{|c|c|c|c|c|c|c|c|c|c|c|}
\hline \multirow[t]{2}{*}{ SNP ID } & \multirow[t]{2}{*}{ Model } & \multirow[t]{2}{*}{ Genotype } & \multicolumn{4}{|c|}{ IS patients with/without HYP } & \multicolumn{4}{|c|}{ IS patients with/without CHD } \\
\hline & & & $\begin{array}{l}\text { With } \\
\text { HYP }\end{array}$ & $\begin{array}{l}\text { Without } \\
\text { HYP }\end{array}$ & $\begin{array}{l}\text { OR } \\
(95 \% \mathrm{Cl})\end{array}$ & $\mathrm{p}$ & $\begin{array}{l}\text { With } \\
\text { CHD }\end{array}$ & $\begin{array}{l}\text { Without } \\
\text { CHD }\end{array}$ & $\begin{array}{l}\text { OR } \\
(95 \% \mathrm{Cl})\end{array}$ & $\mathrm{p}$ \\
\hline \multirow{10}{*}{$\begin{array}{l}\text { ZSCAN25 } \\
\text { rs10242455 }\end{array}$} & Allele & A & 494 & 211 & 1 & & 147 & 558 & 1 & \\
\hline & & G & 186 & 63 & $\begin{array}{l}1.26 \\
(0.91- \\
1.75)\end{array}$ & 0.165 & 59 & 190 & $\begin{array}{l}1.18 \\
(0.84- \\
1.66)\end{array}$ & 0.349 \\
\hline & Genotype & AA & 172 & 84 & 1 & & 49 & 207 & 1 & \\
\hline & & $A G$ & 150 & 43 & $\begin{array}{l}1.72 \\
(1.12- \\
2.64)\end{array}$ & 0.014 & 49 & 144 & $\begin{array}{l}1.4 \\
(0.88- \\
2.21)\end{array}$ & 0.151 \\
\hline & & GG & 18 & 10 & $\begin{array}{l}0.82 \\
(0.36- \\
1.88)\end{array}$ & 0.640 & 5 & 23 & $\begin{array}{l}0.8 \\
(0.28- \\
2.24)\end{array}$ & 0.667 \\
\hline & Dominant & AA & 172 & 84 & 1 & & 49 & 207 & 1 & \\
\hline & & AG-GG & 168 & 53 & $\begin{array}{l}1.55 \\
(1.03- \\
2.32)\end{array}$ & 0.035 & 54 & 167 & $\begin{array}{l}1.31 \\
(0.84- \\
2.05)\end{array}$ & 0.234 \\
\hline & Recessive & $A A-A G$ & 322 & 127 & 1 & & 98 & 351 & 1 & \\
\hline & & GG & 18 & 10 & $\begin{array}{l}0.66 \\
(0.29- \\
1.49)\end{array}$ & 0.315 & 5 & 23 & $\begin{array}{l}0.68 \\
(0.25- \\
1.87)\end{array}$ & 0.458 \\
\hline & $\begin{array}{l}\text { Log- } \\
\text { additive }\end{array}$ & - & - & - & $\begin{array}{l}1.27 \\
(0.90- \\
1.78)\end{array}$ & 0.174 & - & - & $\begin{array}{l}1.13 \\
(0.79- \\
1.63)\end{array}$ & 0.496 \\
\hline \multicolumn{11}{|c|}{$\begin{array}{l}\text { IS; ischemic stroke; HYP, hypertension; CHD, coronary heart disease; SNP, single nucleotide polymorphism; OR, odds } \\
\text { ratio; } 95 \% \mathrm{Cl}, 95 \% \text { confidence interval. }\end{array}$} \\
\hline
\end{tabular}

\section{The relationship between genotypes and blood lipid parameters in IS Patients}

Next, the association of clinical characteristics with different genotypes of ZSCAN25 and CYP2E1 polymorphisms among IS patients was evaluated, as shown in Table 5. The results showed that the rs 10242455 genotype was significantly associated with PLT level $(p=0.004)$. Moreover, the rs2070672-GG carrier had a hemoglobin level $(p=0.031)$ than the AA or AG carrier in IS cases. However, no statistically association was observed between ZSCAN25 and CYP2E1 polymorphisms and serum lipid level, including triglyceride, total cholesterol, HDL-C and LDL-C $(p>0.05)$. 
Table 5

Association of clinical characteristics with different genotypes of ZSCAN25 and CYP2E1 polymorphisms among IS patients

\begin{tabular}{|c|c|c|c|c|}
\hline \multirow[t]{2}{*}{ Characteristics } & \multicolumn{4}{|l|}{ rs10242455 } \\
\hline & AA & AG & GG & p \\
\hline Total protein (g/L) & $52.93 \pm 25.27$ & $53.82 \pm 23.16$ & $56.98 \pm 24.25$ & 0.733 \\
\hline Total bilirubin (mmol/L) & $12.77 \pm 7.95$ & $11.64 \pm 6.40$ & $13.22 \pm 10.47$ & 0.287 \\
\hline Triglyceride (mmol/L) & $1.61 \pm 0.88$ & $1.59 \pm 1.02$ & $1.78 \pm 1.27$ & 0.684 \\
\hline Total cholesterol (mmol/L) & $3.99 \pm 1.01$ & $3.86 \pm 1.04$ & $4.06 \pm 1.11$ & 0.390 \\
\hline HDL-C (mmol/L) & $1.21 \pm 0.32$ & $1.14 \pm 0.31$ & $1.08 \pm 0.28$ & 0.054 \\
\hline LDL-C (mmol/L) & $1.91 \pm 0.59$ & $1.80 \pm 0.62$ & $1.93 \pm 0.61$ & 0.202 \\
\hline Hemoglobin (g/L) & $123.83 \pm 43.91$ & $122.82 \pm 47.08$ & $113.98 \pm 48.65$ & 0.600 \\
\hline $\operatorname{TLC}\left(10^{9} / \mathrm{L}\right)$ & $7.97 \pm 7.84$ & $7.39 \pm 7.73$ & $9.77 \pm 14.09$ & 0.395 \\
\hline $\operatorname{PLT}\left(10^{9} / \mathrm{L}\right)$ & $165.57 \pm 83.92$ & $137.30 \pm 85.76$ & $139.85 \pm 82.36$ & 0.004 \\
\hline \multirow[t]{2}{*}{ Characteristics } & \multicolumn{4}{|l|}{ rs2070672 } \\
\hline & AA & AG & GG & $p$ \\
\hline Total protein $(\mathrm{g} / \mathrm{L})$ & $53.76 \pm 24.23$ & $53.43 \pm 24.38$ & $51.23 \pm 26.57$ & 0.889 \\
\hline Total bilirubin (mmol/L) & $12.54 \pm 8.28$ & $11.67 \pm 6.29$ & $14.43 \pm 6.05$ & 0.207 \\
\hline Triglyceride (mmol/L) & $1.66 \pm 1.06$ & $1.55 \pm 0.80$ & $1.49 \pm 0.64$ & 0.450 \\
\hline Total cholesterol (mmol/L) & $3.95 \pm 1.07$ & $3.92 \pm 0.93$ & $4.03 \pm 1.04$ & 0.876 \\
\hline HDL-C (mmol/L) & $1.18 \pm 0.32$ & $1.17 \pm 0.31$ & $1.18 \pm 0.35$ & 0.959 \\
\hline LDL-C (mmol/L) & $1.87 \pm 0.61$ & $1.85 \pm 0.58$ & $1.89 \pm 0.68$ & 0.898 \\
\hline Hemoglobin (g/L) & $124.94 \pm 43.87$ & $123.17 \pm 45.25$ & $99.46 \pm 56.45$ & 0.031 \\
\hline $\operatorname{TLC}\left(10^{9} / \mathrm{L}\right)$ & $7.60 \pm 6.92$ & $8.57 \pm 10.64$ & $5.89 \pm 2.20$ & 0.263 \\
\hline $\operatorname{PLT}\left(10^{9} / \mathrm{L}\right)$ & $157.97 \pm 85.29$ & $144.65 \pm 85.07$ & $150.42 \pm 90.12$ & 0.325 \\
\hline \multirow[t]{2}{*}{ Characteristics } & \multicolumn{4}{|l|}{ rs2515641 } \\
\hline & TT & $\mathrm{TC}$ & $\mathrm{CC}$ & $\mathrm{p}$ \\
\hline Total protein (g/L) & $46.44 \pm 27.88$ & $53.69 \pm 24.37$ & $53.57 \pm 24.38$ & 0.582 \\
\hline Total bilirubin (mmol/L) & $13.36 \pm 3.85$ & $12.33 \pm 6.73$ & $12.31 \pm 8.10$ & 0.887 \\
\hline Triglyceride (mmol/L) & $1.19 \pm 0.53$ & $1.59 \pm 0.81$ & $1.65 \pm 1.04$ & 0.238 \\
\hline Total cholesterol (mmol/L) & $3.67 \pm 1.04$ & $3.95 \pm 0.95$ & $3.95 \pm 1.06$ & 0.619 \\
\hline
\end{tabular}

SNP, single nucleotide polymorphism; IS; ischemic stroke; HDL-C, high-density lipoprotein cholesterol; LDL-C, lowdensity lipoprotein cholesterol; TLC, total leukocyte count; PLT, platelets.

Bold indicate that $p<0.05$ indicates statistical significance. 


\begin{tabular}{|c|c|c|c|c|}
\hline \multirow[t]{2}{*}{ Characteristics } & \multicolumn{4}{|l|}{ rs10242455 } \\
\hline & AA & AG & GG & $\mathrm{p}$ \\
\hline HDL-C (mmol/L) & $1.22 \pm 0.43$ & $1.18 \pm 0.33$ & $1.17 \pm 0.31$ & 0.846 \\
\hline LDL-C (mmol/L) & $1.64 \pm 0.46$ & $1.85 \pm 0.56$ & $1.88 \pm 0.61$ & 0.356 \\
\hline Hemoglobin $(\mathrm{g} / \mathrm{L})$ & $110.07 \pm 52.74$ & $122.55 \pm 46.52$ & $124.00 \pm 44.38$ & 0.528 \\
\hline $\operatorname{TLC}\left(10^{9} / \mathrm{L}\right)$ & $5.26 \pm 1.81$ & $8.95 \pm 11.09$ & $7.45 \pm 6.71$ & 0.115 \\
\hline $\operatorname{PLT}\left(10^{9} / \mathrm{L}\right)$ & $127.5 \pm 97.06$ & $141.28 \pm 79.16$ & $159.36 \pm 87.90$ & 0.075 \\
\hline \multicolumn{5}{|c|}{$\begin{array}{l}\text { SNP, single nucleotide polymorphism; IS; ischemic stroke; HDL-C, high-density lipoprotein cholesterol; LDL-C, low- } \\
\text { density lipoprotein cholesterol; TLC, total leukocyte count; PLT, platelets. }\end{array}$} \\
\hline \multicolumn{5}{|c|}{ Bold indicate that $p<0.05$ indicates statistical significance. } \\
\hline
\end{tabular}

\section{Discussion}

In this study, we firstly evaluated the relationship between three SNPs (rs10242455 in ZSCAN25, rs2070672 and rs2515641 in CYP2E1) and the risk of IS in a Chinese Han population. Our results displayed that rs 10242455 was associated with a reduced IS susceptibility; while rs2070672 and rs2515641 were correlated with an increased IS occurrence. Gender-stratified analyses showed that rs10242455 and rs2070672 polymorphisms affected IS risk in males but not in females. In addition, rs 10242455 was significantly associated with hypertension in IS patients. These findings suggested that ZSCAN25 and CYP2E1 genetic variants might participate in the etiology of IS.

ZSCAN25 gene is located in human chromosome 7q22.1. The downregulation of ZNF498 increased the malignant proliferation of ovarian cancer ${ }^{[20]}$. Previously, some studies provided evidence for the correlation of ZNF498 polymorphisms with anthropometric traits or disease. Ozren Polašek et.al reported that ZSCAN25 polymorphism was associated with body weight, hip circumference, and brachial circumference ${ }^{[21]}$. In addition, ZSCAN25 polymorphism is known to be related to epilepsy susceptibility ${ }^{[22]}$. However, it is uncertain for the correlation between the ZSCAN25 polymorphism and IS occurrence. Our results displayed that rs10242455 in ZSCAN25 was a protective effect for the decreased risk of IS. The present study firstly provided evidence on the association between ZSCAN25 variants and IS susceptibility.

CYP2E1 gene is located on chromosome 10q26.3. CYP2E1 induction that promotes oxidative damage was reported to be involved in liver damage by induced hyperlipidemia and acute cerebral ischemia/reperfusion [23, 24], suggesting that it might play an important role in IS. CYP2E1 rs3813867 polymorphism was found to be associated with IS risk in the Korean population ${ }^{[18]}$. Similarly, a significant association was observed between $C Y P 2 E 1 \star 5 B$ and the occurrence of stroke in Turkish population [19]. However, the correlation of CYP2E1 variants (rs2070672 and rs2515641) with IS risk has not been reported in previous studies. Here, our results firstly suggested that rs2070672 (g.4682A>G) and rs2515641 (c.1263C > T) in CYP2E1 were risk-effect factor for the susceptibility to IS in the Chinese Han population. Our finding further supported that the CYP2E1 gene might play an important role in pathogenesis of IS.

The mortality rate for IS was increased with age rising, especially over the age of 65 years accounting for two-thirds of IS patients ${ }^{[25,26]}$. Stratified by age, we found that rs 10242455 might be a protective effect on IS susceptibility at age $\leq 64$ years, suggested that the risk association of the polymorphisms might be age dependent. Stroke is a sex-specific disease and the incidence of stroke is higher in men than in women ${ }^{[27]}$. Stratification analysis by sex showed that the contribution of rs10242455 to the reduced IS risk and rs2070672 to the increased IS occurrence in males but not females, 
which indicated that the influence of genetic factors on IS risk might present gender difference. Han TS et al. reported hypertension is a conventional risk factor for stroke, and stroke patients could be saved with appropriate management of hypertension [28]. Given that hypertension is the risk factor of IS, we evaluated the association of ZSCAN25 and CYP2E1 variants with hypertension in IS patients. Our results displayed that rs10242455 was significantly associated with hypertension in IS patients. In addition, the correlation of these variants with CHD in IS patients was further investigated. However, no significant association was observed. These results are needed to affirm in a larger sample set.

Our study provided some interesting findings that ZSCAN25 and CYP2E1 polymorphisms might affect the occurrence of IS, but there were several potential limitations. First, our study was a hospital-based case-control study based on a Chinese Han population, so the selection bias could be unavoidable and the results might not apply to other populations. Second, data of some IS risk factors (e.g., smoking, alcohol and BMI) was insufficient which limited our further analysis of the gene-environment interaction. Third, functional studies were needed to elucidate the biologic mechanisms of ZSCAN25 and CYP2E1 polymorphisms on IS.

\section{Conclusion}

In conclusion, we firstly found that rs10242455 in ZSCAN25, rs2070672 and rs2515641 in CYP2E1 were associated with the occurrence of IS in a Chinese Han population. Our finding increased our knowledge regarding the effect of ZSCAN25 and CYP2E1 gene on the process of IS, and also provided some data for future explorations of the relationship between ZSCAN25 and CYP2E1 and IS risk in different populations. However, well-designed multicenter studies are needed to verify our results.

\section{Declarations}

\section{Ethics approval and consent to participate}

This study protocol was in accordance with the Declaration of Helsinki and permitted by the ethics committee of The Second Affiliated Hospital of Xi'an Jiaotong University, and written informed consent was obtained from all subjects.

\section{Consent for publication}

Not applicable.

\section{Availability of data and material}

All the data regarding the findings are available within the manuscript. Anyone who is interested in the information should contact the corresponding author.

\section{Competing interests}

The authors declare that they have no conflict of interest.

\section{Funding}

This work is supported by General Key Projects of Shaanxi Province (No: 2018SF-125). 


\section{Author Contributions}

The work presented here was carried out in collaboration between all authors. Haozheng Yuan carried out the molecular genetic studies and drafted the manuscript. Pei Fan and Li Yao designed the methods and experiments, performed the statistical analyses and interpreted the results. Yuying Lv designed primers and performed the SNP genotyping experiments. Haidong Wei and Juan Zheng collected clinical information about patients and performed the SNP genotyping experiments. Xinsheng Han conceived of the study, worked on associated data collection and their interpretation, participated in the design and coordination of the study, and funded the study. All authors read and approved the final manuscript.

\section{Acknowledge}

The authors want to thank the participants in the study.

\section{References}

1. Feigin VL, Norrving B, Mensah GA (2017) Global Burden of Stroke. Circulation research 120 (3):439-448. doi:10.1161/circresaha.116.308413

2. Zhou M, Wang H, Zeng X, Yin P, Zhu J, Chen W, Li X, Wang L, Wang L, Liu Y, Liu J, Zhang M, Qi J, Yu S, Afshin A, Gakidou E, Glenn S, Krish VS, Miller-Petrie MK, Mountjoy-Venning WC, Mullany EC, Redford SB, Liu H, Naghavi M, Hay SI, Wang L, Murray CJL, Liang X (2019) Mortality, morbidity, and risk factors in China and its provinces, 1990-2017: a systematic analysis for the Global Burden of Disease Study 2017. Lancet (London, England) 394 (10204):11451158. doi:10.1016/s0140-6736(19)30427-1

3. Cabral NL, Freire AT, Conforto AB, Dos Santos N, Reis FI, Nagel V, Guesser VV, Safanelli J, Longo AL (2017) Increase of Stroke Incidence in Young Adults in a Middle-Income Country: A 10-Year Population-Based Study. Stroke 48 (11):2925-2930. doi:10.1161/strokeaha.117.018531

4. Wang W, Jiang B, Sun H, Ru X, Sun D, Wang L, Wang L, Jiang Y, Li Y, Wang Y, Chen Z, Wu S, Zhang Y, Wang D, Wang Y, Feigin VL (2017) Prevalence, Incidence, and Mortality of Stroke in China: Results from a Nationwide PopulationBased Survey of 480687 Adults. Circulation 135 (8):759-771. doi:10.1161/circulationaha.116.025250

5. Yu J, Luo D, Zhou F, Jin T, Liang X, Yu D (2017) Polymorphism of TNIP1 was associated with atherosclerotic ischemic stroke in southern Han Chinese but unrelated with telomere. Int J Clin Exp Pathol 10 (3):3510-3516

6. Niu F, Wei B, Yan M, Li J, Ouyang Y, Jin T (2018) Matrix metalloproteinase-2 gene polymorphisms are associated with ischemic stroke in a Hainan population. Medicine 97 (39):e12302. doi:10.1097/md.0000000000012302

7. Li G, Zhu L, Bai M, Wang L, Yuan D, He Y, Jin T (2018) The impact of the AHSG genetic polymorphism on the risk of ischemic stroke: a case-control study. INTERNATIONAL JOURNAL OF CLINICAL AND EXPERIMENTAL PATHOLOGY 11 (10):5094-5100

8. Laity JH, Lee BM, Wright PE (2001) Zinc finger proteins: new insights into structural and functional diversity. Current opinion in structural biology 11 (1):39-46. doi:10.1016/s0959-440x(00)00167-6

9. Seidel K, Kirsch S, Lucht K, Zaade D, Reinemund J, Schmitz J, Klare S, Li Y, Schefe JH, Schmerbach K, Goldin-Lang P, Zollmann FS, Thone-Reineke C, Unger T, Funke-Kaiser H (2011) The promyelocytic leukemia zinc finger (PLZF) protein exerts neuroprotective effects in neuronal cells and is dysregulated in experimental stroke. Brain pathology (Zurich, Switzerland) 21 (1):31-43. doi:10.1111/j.1750-3639.2010.00427.x

10. Ding H, Xu Y, Bao X, Wang X, Cui G, Wang W, Hui R, Wang DW (2010) Confirmation of genomewide association signals in Chinese Han population reveals risk loci for ischemic stroke. Stroke 41 (1):177-180. 
doi:10.1161/strokeaha.109.567099

11. Yu J, Zhou F, Luo D, Wang N, Zhang C, Jin T, Liang X, Yu D (2017) ZNF208 polymorphisms associated with ischemic stroke in a southern Chinese Han population. The journal of gene medicine 19 (1-2). doi:10.1002/jgm.2937

12. Schneider E, Clark DS (2013) Cytochrome P450 (CYP) enzymes and the development of CYP biosensors. Biosensors \& bioelectronics 39 (1):1-13. doi:10.1016/j.bios.2012.05.043

13. Huang H, Al-Shabrawey M, Wang MH (2016) Cyclooxygenase- and cytochrome P450-derived eicosanoids in stroke. Prostaglandins \& other lipid mediators 122:45-53. doi:10.1016/j.prostaglandins.2015.12.007

14. Cheng J, Krausz KW, Li F, Ma X, Gonzalez FJ (2013) CYP2E1-dependent elevation of serum cholesterol, triglycerides, and hepatic bile acids by isoniazid. Toxicology and applied pharmacology 266 (2):245-253.

doi:10.1016/j.taap.2012.10.024

15. Chang LS, Hsu YW, Lu CC, Lo MH, Hsieh KS, Li SC, Chang WC, Kuo HC (2017) CYP2E1 Gene Polymorphisms Related to the Formation of Coronary Artery Lesions in Kawasaki Disease. The Pediatric infectious disease journal 36 (11):1039-1043. doi:10.1097/inf.0000000000001657

16. Xu J, Peng Y, Zeng Y, Hua YQ, Xu XL (2019) 2, 3, 4', 5-tetrahydroxystilbene-2-0-beta-d Glycoside Attenuates Age- and Diet-Associated Non-Alcoholic Steatohepatitis and Atherosclerosis in LDL Receptor Knockout Mice and Its Possible Mechanisms. International journal of molecular sciences 20 (7). doi:10.3390/ijms20071617

17. Nosti-Palacios R, Gomez-Garduno J, Molina-Ortiz D, Calzada-Leon R, Dorado-Gonzalez VM, Vences-Mejia A (2014) Aspartame Administration and Insulin Treatment Altered Brain Levels of CYP2E1 and CYP3A2 in StreptozotocinInduced Diabetic Rats. International journal of toxicology 33 (4):325-331. doi:10.1177/1091581814540480

18. Kim SK, Yim SV, Lee BC (2012) Association between cytochrome P450 promoter polymorphisms and ischemic stroke. Experimental and therapeutic medicine 3 (2):261-268. doi:10.3892/etm.2011.388

19. Turkanoglu Ozcelik A, Can Demirdogen B, Demirkaya S, Adali O (2017) Association of cytochrome P4502E1 and $\mathrm{NAD}(\mathrm{P}) \mathrm{H}$ :quinone oxidoreductase 1 genetic polymorphisms with susceptibility to large artery atherosclerotic ischemic stroke: a case-control study in the Turkish population. Neurological sciences : official journal of the Italian Neurological Society and of the Italian Society of Clinical Neurophysiology 38 (6):1077-1085. doi:10.1007/s10072017-2930-9

20. Yamanoi K, Baba T (2019) Acquisition of a side population fraction augments malignant phenotype in ovarian cancer. Sci Rep 9 (1):14215. doi:10.1038/s41598-019-50794-w

21. Polasek O, Marusic A, Rotim K, Hayward C, Vitart V, Huffman J, Campbell S, Jankovic S, Boban M, Biloglav Z, Kolcic I, Krzelj V, Terzic J, Matec L, Tometic G, Nonkovic D, Nincevic J, Pehlic M, Zedelj J, Velagic V, Juricic D, Kirac I, Belak Kovacevic S, Wright AF, Campbell H, Rudan I (2009) Genome-wide association study of anthropometric traits in Korcula Island, Croatia. Croatian medical journal 50 (1):7-16. doi:10.3325/cmj.2009.50.7

22. Al-Eitan LN, Al-Dalalah IM, Mustafa MM (2019) Genetic polymorphisms of CYP3A5, CHRM2, and ZNF498 and their association with epilepsy susceptibility: a pharmacogenetic and case-control study. Pharmacogenomics and personalized medicine 12:225-233. doi:10.2147/pgpm.s212433

23. Gong WH, Zheng WX, Wang J, Chen SH, Pang B, Hu XM, Cao XL (2012) Coexistence of hyperlipidemia and acute cerebral ischemia/reperfusion induces severe liver damage in a rat model. World journal of gastroenterology 18 (35):4934-4943. doi:10.3748/wjg.v18.i35.4934

24. Cao XL, Du J, Zhang Y, Yan JT, Hu XM (2015) Hyperlipidemia exacerbates cerebral injury through oxidative stress, inflammation and neuronal apoptosis in MCAO/reperfusion rats. Experimental brain research 233 (10):2753-2765. doi:10.1007/s00221-015-4269-x

25. Global, regional, and national incidence, prevalence, and years lived with disability for 354 diseases and injuries for 195 countries and territories, 1990-2017: a systematic analysis for the Global Burden of Disease Study 2017 (2018). 
Lancet (London, England) 392 (10159):1789-1858. doi:10.1016/s0140-6736(18)32279-7

26. Krishnamurthi RV, Feigin VL, Forouzanfar MH, Mensah GA, Connor M, Bennett DA, Moran AE, Sacco RL, Anderson

LM, Truelsen T, O'Donnell M, Venketasubramanian N, Barker-Collo S, Lawes CM, Wang W, Shinohara Y, Witt E, Ezzati M, Naghavi M, Murray C (2013) Global and regional burden of first-ever ischaemic and haemorrhagic stroke during 1990-2010: findings from the Global Burden of Disease Study 2010. The Lancet Global health 1 (5):e259-281. doi:10.1016/s2214-109x(13)70089-5

27. Poorthuis MH, Algra AM, Algra A, Kappelle LJ, Klijn CJ (2017) Female- and Male-Specific Risk Factors for Stroke: A Systematic Review and Meta-analysis. JAMA neurology 74 (1):75-81. doi:10.1001/jamaneurol.2016.3482

28. Han TS, Wang HH, Wei L, Pan Y (2017) Impacts of undetected and inadequately treated hypertension on incident stroke in China. BMJ open 7 (10):e016581. doi:10.1136/bmjopen-2017-016581

\section{Supplementary Files}

This is a list of supplementary files associated with this preprint. Click to download.

- SuppTables.docx 